\title{
Smart Parking Systems: Reviewing the Literature, Architecture and Ways Forward
}

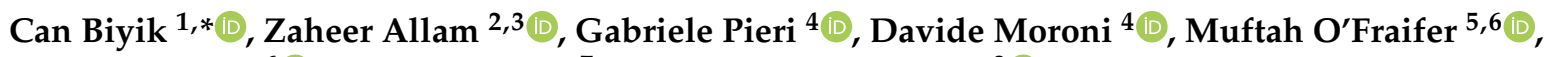 \\ Eoin $\mathrm{O}^{\prime}$ Connell ${ }^{6}{ }^{(\mathbb{C}}$, Stephan Olariu ${ }^{7}$ and Muhammad Khalid ${ }^{8}$ (i)
}

1 Faculty of Engineering and Natural Sciences, Ankara Yildirim Beyazit University, 06760 Ankara, Turkey

2 Sorbonne Business School, Université Paris 1 Panthéon-Sorbonne, 75013 Paris, France; zaheer.allam@chaire-eti.org

3 School of Architecture and Built Environment, Deakin University, Geelong, VIC 3220, Australia

4 National Research Council, Institute of Information Science and Technologies, 56124 Pisa, Italy; gabriele.pieri@isti.cnr.it (G.P.); davide.moroni@isti.cnr.it (D.M.)

5 Electronic Engineering Department, The College of IndustriaL Technology—CIT, Misurata 2478, Libya; muftah.fraifer@mail.com

6 Department of Electronic \& Computer Engineering, University of Limerick, V94 T9PX Limerick, Ireland; Eoin.OConnell@ul.ie

7 Department of Computer Science, Old Dominion University, Norfolk, VA 23529, USA; olariu@cs.odu.edu

8 Computer Science, Northumbria University, Newcastle upon Tyne NE1 8ST, UK;

m.khalid@northumbria.ac.uk

* Correspondence: cbiyik@ybu.edu.tr

Citation: Biyik, C.; Allam, Z.; Pieri, G.; Moroni, D.; O'Fraifer, M.;

O'Connell, E.; Olariu, S.; Khalid, M. Smart Parking Systems: Reviewing the Literature, Architecture and Ways Forward. Smart Cities 2021, 4, 623-642. https://doi.org/10.3390/

smartcities 4020032

Academic Editor: Luis Francisco Díez

Received: 24 March 2021

Accepted: 22 April 2021

Published: 28 April 2021

Publisher's Note: MDPI stays neutral with regard to jurisdictional claims in published maps and institutional affiliations.

\begin{abstract}
The Internet of Things (IoT) has come of age, and complex solutions can now be implemented seamlessly within urban governance and management frameworks and processes. For cities, growing rates of car ownership are rendering parking availability a challenge and lowering the quality of life through increased carbon emissions. The development of smart parking solutions is thus necessary to reduce the time spent looking for parking and to reduce greenhouse gas emissions. The principal role of this research paper is to analyze smart parking solutions from a technical perspective, underlining the systems and sensors that are available, as documented in the literature. The review seeks to provide comprehensive insights into the building of smart parking solutions. A holistic survey of the current state of smart parking systems should incorporate the classification of such systems as big vehicular detection technologies. Finally, communication modules are presented with clarity.
\end{abstract}

Keywords: smart parking systems; architecture; layers; IoT; smart cities

\section{Introduction}

The emergence of automobiles in the transportation sector brought about unprecedented changes, including increased flexibility in travel and the movement of goods, as well as the growth of various economic sectors. However, automobiles have also given rise to some notable challenges that have gradually reached a level requiring urgent solutions. Such challenges include environmental degradation, emissions, and noise. Additionally, people and animals are exposed to harm, with increased road accidents as more cars are introduced onto the roads $[1,2]$. Furthermore, automobiles have also contributed to economic issues associated with traffic jams that are now rampant in most cities. Automobiles have gradually come to pose a challenge to city planners, especially in terms of ensuring that the increasing influx of automobiles may be accommodated, through both the construction of roads and the creation of enough parking spaces [3-5].

The challenge of parking is particularly important, as most people prefer private car ownership, something that is deeply ingrained in the daily routines of many of us [6]. For this reason, the search for a parking spot in busy towns and cities is a daunting 
endeavor, leading to time wastage and unwarranted consumption of fuel, and, importantly, contributing to climate change $[7,8]$. The issue of parking is significant to the point that it has been raised in discussions of climate change mitigation as well as in political arenas $[9,10]$.

In the new era, where technology has been accepted as one of the most critical tools for solving some of the challenges faced in the 21st century, especially in urban areas, the issue of parking has not been left behind. With the adoption of the Smart City model in most urban areas, smart digital solutions have emerged. Among them is the smart parking system, which, as argued by Naphade et al. [11], may be instrumental in bringing order and sanity to parking lots. According to them, the smart parking system could be customized to combine both technology and human innovations in order to optimize the utilization of scarce resources such as fuel, time, and space. In this way, as noted by Babic et al. [12], urban areas can benefit from achieving faster, easier, and denser parking of vehicles by ensuring every available parking slot is utilized efficiently. Overall, the smart parking system is expected to help urban management reduce traffic and lower parking management costs, among others.

Borgonovo et al. [13] suggested that smart parking systems are set to bring positive changes to different urban stakeholders, who, in one way or another, shoulder the burden of haphazardly managed parking spaces. For instance, it is believed that these systems will have a positive impact on traffic accident rates, i.e., crashes resulting from driver attention deficits as they concentrate on searching for parking spaces or rush to occupy existing ones [13]. A decline in accidents will coincide with speed and efficiency in locating vacant spaces by drivers via smartphone apps; hence, drivers will no longer need to scramble for spaces [14]. Besides helping drivers, smart parking systems are expected to help parking facility managers and owners to maximize the utilization of available spaces and resources in a way that increase their revenue as well as improving the parking experience of their clients [15]. For instance, Sajeev et al. [16] noted that the use of a smart system would allow managers to set parking fees commensurate to the facility's current occupancy. Therefore, these parking systems will have both financial and logistical merits for owners, political leaders, and urban managers.

Another category of stakeholder that would benefit from the implementation of smart parking systems is traffic law enforcement. With such systems, it would be possible to introduce a practical framework that would allow the identification of real-time cases of density violation and illegal or improper parking [17]. Smart parking systems would also be able to determine peak times of parking violations and, hence, facilitate the implementation of countermeasures. The large amount of data that would be generated from the different Internet-of-Things (IoT) devices and sensors that would be located in different spots is expected, at the very least, to help in transforming urban parking into more sustainable zones, especially in terms of reducing pollution by cutting driving time, thereby reducing traffic in streets and ensuring the proper utilization of resources in parking lots [18]. The net effect thereafter would be improved livability with fewer health complications and more sustainable environmental practices [19].

The remaining section of the present research is organized as follows: The second section is dedicated to exploring the existing architecture of smart parking systems based on the deployment and implementation of various developments, including the application layer, network layer, transaction layer, and physical layer. This is qualified in Section 3 by providing a brief introduction to the physical deployment of such systems in various cities. Next, the fourth section presents a critical analysis of the technology for smart parking systems as reported in the relevant literature. Section 5 is a proposal for research into the identification of the top and most novel trends related to sensors, software solutions, and networking via the application of a descriptive research methodology.

\section{The Architecture of Smart Parking Systems}

A smart parking system is an architectural framework that comprises different application platforms integrated into embedded systems. For instance, reserved parking 
spaces allow users to request the application layer, wherein the request will immediately be processed through a network layer [20]. As a way of handling the user request, parking providers are expected to utilize the network layer to process the interaction with the transaction layer, as explained by Kayal and Perros [21]. Finally, the transaction layer's consensus mechanism protocol and the individual parking provider update the distributed ledger.

Ahmed et al. [22] explained that the smart parking solution architecture is majorly represented by four components: the application layer, network layer, transaction layer, and physical layer. An illustration of the layered architecture is provided in Figure 1, which presents the instrumental aspects of the systems. The details of the smart parking subsystems are explained as follows.

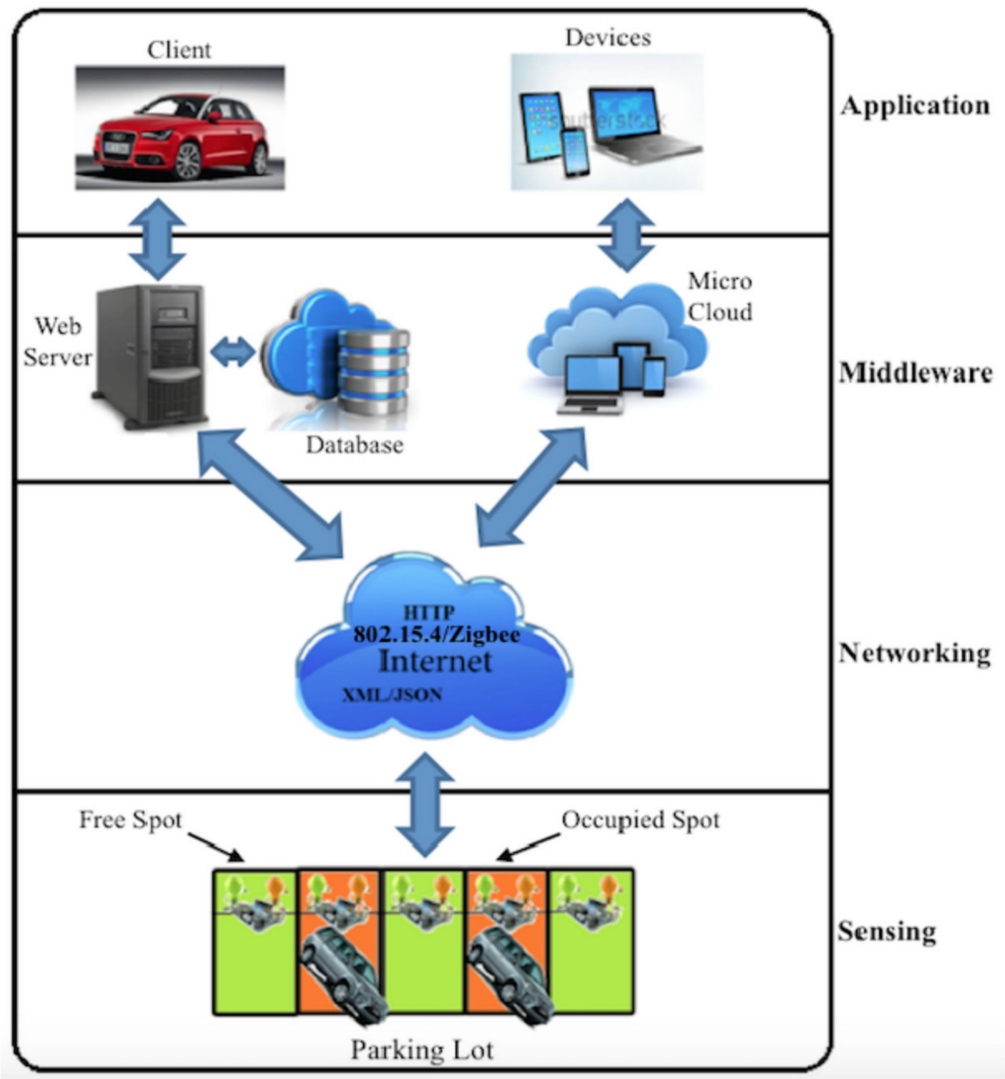

Figure 1. Layer architecture for integrated smart parking systems. Adapted from Ahmed et al. [22].

\subsection{Application Layer}

The application layer is the top layer of the architecture stack that allows the participants to interact with the system that they use, the mobile application (i.e., Android and iOS), or the Web application. Here, as highlighted by Yang et al. [23], users are capable of searching for their preferred parking locations, and they can make reservations. Similarly, the parking services provider can send parking-related information, e.g., parking space availability, to the providers and the offers to the integrated systems. Since the users interact with the integrated system directly, the layer delivers the end-users' final service.

\subsection{Network Layer}

The network layer ensures seamless communication among the various parking centers, integrated systems, and users. The user and parking center data are transmitted to the integrated system through a layer. The layer contains the different types of communication technologies that may include LAN and WAN, which are used by the users, parking service providers, and the IoT devices related to the parking systems (e.g., the parking sensors and the security camera). They may contain different wireless technologies that 
include Bluetooth, WI-FI, etc., which, along with the existing GSM technologies, exist as $4 \mathrm{G}$ and $5 \mathrm{G}$ [20].

\subsection{Transaction Layer}

This is the layer that is mandated to transact the nodes in the network. The users and the various parking centers exchange the data more securely through the smart contract and the consensus mechanisms. The parking center also updates the public ledger through the layer. The transaction layer preserves the transparent quality of the transaction and the security of the data transmission without trusted third parties, especially if they rely on Blockchain systems, which are immutable [24].

\subsection{Physical Layers}

The physical layers deal specifically with the mechanisms and the electronic anchorage of the system. The physical layer is based on the set of the physical sensors and the data received from the entities collected that are analyzed and used to manage the entities. The different types of sensors are the significant elements of the layer. The use of IoT device sensors can be recognized through the availability, which can be identified from the physical layers as expressed by [22].

\section{Examples of Best Practices in Smart Parking Systems}

There exist various practices in the global smart parking scene. The intelligent parking architectures that are discussed in this section present the universal sleek parking case as carried out in Barcelona, Bussan Riga, Santander, and Valletta. The overview underpins the various physical communication technologies specific to smart parking while spotting the major aspects that influence intelligent parking performance.

Barcelona, a Smart City, has achieved a wide range of the merits through investments in IoT for urban systems, including smart parking technology [25]. Sotres et al. [26] explain how city management has invested in deploying sensors system for motorists to guide them to e-parking spots. A total of 600 wireless parking sensors were deployed on the streets of Barcelona, les Cortes district, in 2014 [26]. The embedded aspects were placed underneath the asphalt, and the sensors were then used to identify the available parking spaces and notify the motorists. The program was intended to cut down on emissions and congestion by providing motorists real-time direction on the availability and location of open parking spaces. Access to sensor data was conducted through the proprietary application programming interface (API) of varying technology vendors operating in the smart parking space.

Another case scenario is Busan city, South Korea, where IoT technology was utilized as part of the first-generation IoT-enabled smart city pilot project [27]. The proposed intelligent parking services were enhanced annually between 2015 and 2017. In the first year (2015), the parking sensors were fixed to public parking lots to provide real-time parking service data. In the following year, closed-circuit television (CCTV)-based image recognition technology was implemented to gain better insights into the occupancy data. Finally, in the final year (2017), parking spaces with electronic vehicle charging stations were incorporated. Six indoor ones were selected to be part of the smart parking use in the wiseIoT project for 2014 intelligent parking sensors. They provide real-time occupancy data in every parking location. The universal grading is proposed by the wise-IoT framework for the platform's interoperability [26].

Another case is that of Riga city, which has paid parking services. The city has an underground parking space for around 167 vehicles, which are managed through an installed, automated parking ticketing machine located at the parking's entrance and exit, where drivers acquire a ticket with a QR code with time stamps [28]. The success of this system is such that the vehicle that exceeds the parking time limit will not be capable of parking without being surcharged with additional parking time. 
The city of Santander in Spain also experimented on the implementation of a smart parking solution, with parking lots with built-in inductive sensors. Here, over 250 outdoor parking sensors were installed in the city's primary parking centers to detect the availability of parking spaces [29]. The advantage of these sensors is that they are buried under asphalt, and they work based on ferromagnetic detection. An API was created for the utilization in the exchange of information through the sensors and the client, where data that are collected from the devices therein are then dissipated to the manufacturers back in real time, and they are finally processed and relayed back in the form of free occupied events per parking spot. Interestingly, this framework is also utilized in the municipality for traffic management tasks and the control of traffic lights [30].

In the city of Pisa in Italy, extensive tests for innovative parking solutions have been conducted, and their results have been analyzed. The analysis covered mainly long-/medium-term parking lots mostly used by commuters and was also focused on the different typologies of parking spaces existing, e.g., regular, disabled-reserved, and e-vehicle-reserved. This analysis was performed using autonomous systems based on wireless smart cameras [31], which locally acquire and analyze images while only propagating numeric results toward the centralized server. The objectives were different: having an autonomous intelligent system using energy harvesting through photovoltaic panels; providing a Web platform and mobile applications; and evaluating and validating the design while considering integration with the existing traffic management system. These applications' primary purpose is to integrate collected data from the developed wireless sensors and to transmit the aggregated results to a higher hierarchical level [32].

Another city that offers a practical observation on how the smart parking systems work is the city Valletta, which offers an interesting scenario, as it is walled and has a limited parking infrastructure. Therefore, the accessibility to the city by any vehicle is at the limit of road pricing, amount of pedestrians, and the relativity of parking spaces. The parking system here was introduced to provide real-time parking information and the management of the supply of parking spaces according to the users' demand. However, with the increasing number of vehicles in the city, there is a proposal to introduce a new (smart) parking management system that may help to overcome escalating vehicular challenges. In line with this, there is a plan to install around sixty sensors and a number of cameras as a way of testing and piloting various technologies prior to identifying the most appropriate method for the future upscaling of the City of Valletta [26].

Similarly, the city of Lucca (Italy) is a medieval walled city with comparable issues; in this case, a network of smart cameras able to monitor one or more parking areas of variable dimensions has been set-up. In particular, an infrastructure of light, customizable smart cameras equipped with computer vision logic can evaluate the monitored parking area's occupancy level and identify free or occupied spaces. The presented software architecture is based on a state-of-the-art convolutional neural network, achieving an error rate of 0:4\%. Another interesting and important feature in this study case is that caused by cultural heritage constraints of the historical center of this medieval city; in some spots, a wired electrical network is not present and cannot be arranged, and, in this case, an energy harvesting unit is combined with a custom-designed, very low consumption, embedded vision board running lightweight image processing with a slightly higher error rate of $0: 65 \%$. In both cases, image processing is performed aboard the smart cameras themselves, so no image transmission is required [33].

\section{Related Works}

When it comes to smart parking systems, application layers are the core of processing information collected by sensors and are essential to the dissemination of results to other systems' end-users. However, all the other components of the system are equally important and play a critical role in the handling of all the data and information. For instance, the system encompasses information ranging from registration, storage, and built prediction systems that ameliorate the allocation and the reservation of parking spaces [22]. All of 
these components are integrated into an application that could be accessed via the Web or through smartphones, with the main objective being that of presenting viable solutions to packing challenges. These allow users to perform specific tasks that include reserved parking spaces and finding the nearest parking lots in unique daytime. The data reside in the application layers, and they represent useful tools that are majorly employed in the government's work to improve urban development mobility [23].

Application layers are instrumental as they could help to sustain the information that is of benefit to urban dwellers and cities at large. In parking systems, these layers serve as information centers that feed the cloud-based service. They also serve as IoT management centers, whose roles are the administration of the smart parking system through an IoT integrated service portal. This is demonstrated by Tsai et al. [34], who engineered a cloud-based smart parking system through the Global Positioning System (GPS). This application targets the coordination and provision of data on issues such as the number of vehicles, the availability of free parking spaces in various locations, and the distance between parking spaces. The application could also help in calculating the costs of the parking requests of drivers. Another example is the study of Mainetti et al. [35], who mapped out a smart parking system through an integrated ultra-high frequency (UHF), wireless sensor network (WSN) with RFID technologies. Their system is made up of software features that assemble the parking slot occupancy. They were engineered into an app that navigates drivers into the nearest parking spot. The app also allows users to make parking payments through a near-field communication (NFC)-based wallet system. It makes use of Java REST, APIs, and Google Cloud messaging, which are installed through a principle server for the management of alerts (such as the expiration of the bought time and the state of the booked space), which are prompted to signal traffic cops.

To ensure that different emerging parking systems are crafted to achieve the intended objectives, there exists an IoT cloud-based car parking framework, which was articulated by Ji et al. [36]. This framework consists of a layer of sensors, a communication layer, and an application layer. The proponents of this system utilize the OSGi-based Web application that provides signs for the driver with information on the best available car parking system. The system utilizes various protocols that are perceived to have rendered the system bulky and complicated [36]. Kotb et al. [37] proposed the implementation of the smart parking system as a means of alleviating packing challenges in urban areas. Their proposed system was developed on the basis of ZigBee technology, which is a development of the information of other servers through a gateway, and the server is a subsequent result of the updates on the database. For the individuals who are looking for free parking spaces, the application layer of the structure accesses the available information regarding the parking base through the Internet and collects the scattered parking spot availability information through the use of Web services that pass the information on to motorists. There is a simple application that does not make any consideration of complex problems such as traffic congestion, navigation, and the anticipated availability of parking spots.

Networking protocols are the core part of the information channel from the sensors to the smart parking structure. They can be disordered and interconnected on a particular sensor in the place of the retrieval of data. In smart parking solutions, there could be two different networking frameworks. First, there are cases in which the sensors and users can utilize the same protocol. However, the user protocol utilizes more power, and it needs to have an Internet connection to work efficiently. For example, to access or reserve a particular parking spot, the Internet is required as it is what provides real-time information on such [38]. The parking lot information in the database is dynamic and is updated to provide users with the real-time information. Another case scenario that justifies the need for Internet connectivity is the need to have specific details about the drivers who occupy a given parking space. Without the Internet, such could not be updated automatically, hence, making automatic identification difficult. In such cases, the customer is expected to visit the service providers' website through Bluetooth connectivity. Thereafter, they have to verify their car ownership, a procedure which is considered inconvenient to users. The 
alternative to this extensive Internet requirement is the adoption of sensor protocols that are indirectly connected to the Internet; hence, they have to go through the gateway numerous times. The gateway is in control of the translation of the wireless IoT protocols to the TCP/IP basis. In the face of smart parking solution grievances, the architectural network infrastructure module has to anchor hundreds of thousands of gadgets that are connected and transmitted at every particular interval. They are network executions that have to focus on the deployment of the IoT protocols and the mesh networks that safeguard larger spaces and allow for the transition of the sensor, even when there the gateway nodes fail.

Shi et al. [39] are among researchers that have designed and implemented a parking system on the basis of the ZigBee wireless sensor network. These researchers made a proposal of a smart parking system through the utility of the ZigBee wireless network protocol as the core technology for the communication between the parking monitoring framework and the microcontrollers in the car park. Khanna and Anand [19] note that this system is cheap and has fast adaptation to the lower energy consumption in comparison to other systems. Additionally, it can be executed in the indoor car park, including car parks with several floors. In the work of Wang and He [14], a system that assisted in providing information on the parking and reservation services that are available to the consumers was built.

A sensor network employs ZigBee 1 modules, and it is espoused in a way that monitors the occupancy status of the parking spaces in real time. Every module has luminosity in the sensing vibration that is utilized in the detection of vehicles and Bluetooth connectivity that is achieved with communication of users; smartphone through the Internet. On the basis of the parking spaces, the system can make an analysis of the parking occupancy, determine the parking prices, and notify the users of the prices.

Leone et al. [31] have taken advantage of wireless sensor networks' additional features by proposing a fog computing solution. Indeed, they note that due to embedded sensors' growing computing capabilities, the intelligence can be transferred from the core components (servers and cloud-based facilities) to the border (analysis and aggregation of edge computing data on local gateways). This approach may be pushed further until reaching the devices (the fog computing approach), thus spreading the whole system's intelligence among all architectural elements in a so-called computational continuum. These ideas are validated by the development of an IoT middleware, implemented on top of Contiki and using an IPv6 over Low-Power Wireless Personal Area Networks (6LoWPAN; see Shelby and Bormann's [40] protocol. Its unique feature is the presence of a resource processing engine capable of performing in-network event composition [41]. In this way, the resulting wireless sensor network is capable of fusing data from multiple, possibly low fidelity, sensors that transmit data regarding individual spaces in a parking lot. Due to its in-network event composition, the network itself can conduct a fault-tolerant robust analysis of slot occupancy status.

Other layers that are part of the parking system are the transaction layers, which encompasses cryptographic protocols that are meant to provide communication security to the computer network. The transaction layer protocols are also intended to provide privacy and data integrity between two or more communicating computer applications. These layers are carefully integrated such that they are able to provide additional privacy-related features that include forward secrecy, making sure that there is future disclosure of the encryption keys that cannot be utilized in the decryption of any transaction layers that recommunicated in the past.

Chatzigiannakis et al. [42] researched the instrumental cryptographically founded confidential preservation of smart parking spaces. In their research, they called this instrument the elliptic curve cryptograph, which they argued was an attractive alternative for the resource constrain services that other public key cryptographies were unable to solve. In their system, the authors achieved the privacy concept through the use of zero knowledge roofs that ensured avoidance of the exchange of confidential information and the evaluation of the performance of the studied system and the time executed. They also 
majorly focused on the preservation of privacy in the smart parking system instead of only concentrating on providing parking recommendations. They managed this through the use of various technologies that were used in identifying and locating the disclose users. Thus, a person could allege that the above-mentioned systems were improved in terms of the structures of security and the protection of privacy of the users through the employment of renowned identity protocols and crypto systems.

The application and imitation of the current I-based Internet security protocols and security protection were suitable in the context of the IoT infrastructures as analyzed by Yaqoob et al. [43]. The examination indicated that public cryptography was suitable for IoT systems, provided that the related asymmetric mechanisms were optimized property. Additionally, asymmetric techniques were suitable for the distributed solutions that were proposed since they were effective in addressing the points of key distribution in the settings.

An intelligent smart parking system is not achievable without incorporation of IoT technologies. These technologies provide advanced opportunities that enable stakeholders of smart parking systems to monitor real-time parking scenarios, nearest parking spots, and free parking spot alerts on smartphones in parking systems [44]. As IoT infrastructures are being deployed, there are diverse smart city services that are commonly integrated and, in some instances, this has led and raised concerns on issues related to privacy and trust $[45,46]$. Data privacy and ingenuity, control of the access to the IoT network, and confidentiality and trust among consumers, as well as the enforcement of security and privacy statutes, are among some of the teething problems that smart city proponents have to deal with. There are various degrees of communication stacks that are incorporated with the vast differences of the embedded hardware aspects that could make traditional security countermeasures complicated when directly applied in IoT domains. Recently, ITS systems have taken over IoT technologies, and they managed parking systems that use sensors and cameras in the monitoring of real scenarios with wireless devices [47].

In 2015, Postigo et al. [48] estimated the parking area status through background subtraction and transience map examination. The methodology they used is capable of handling scenarios where there are multiple moving objects, various illumination conditions and occlusions of vehicles, and static surveillance cameras. The researchers concluded that the utility of personal sensors in the monitoring of the parking spaces is expensive and not scalable. Part of the reasons they gave is that the areas that are covered by the surveillance cameras that are in control increase the costs of installation and power consumption, and the long-term sustenance fees of cameras are quite large. Furthermore, cameras require consistent maintenance to ensure that they are functioning, for instance, the removal of dirt that may interfere with the capture of clear images. Some conditions that impact performance include heavy rain and fog. Fraifer and Fernström [49] in their work proposed a parking system that involves the CCTV camera in the monitoring of the areas of the park by dissipating the video to the interconnected system, which utilizes the algorithm for computer vision in the detection of the car on the slot. If there were no cars that were parked on the spot, then there would be a free consideration, which would be occupied. The system would then send the status to all of the server spots. The driver can therefore see the status and then find a free space through the use of the smart phone. The authors reviewed their systems, which helped them craft a discussion on the problems and the demerits of the system. Their discussion converged on the understanding that there are some issues that require to be improved, especially regarding the way that the system detects the status of the spots, as well as how it sends the information on to the server. For instance, the system has to be interconnected to the Internet just in case the connection is slow, which renders it useless. The other issue is that the system requires to be continually connected to a power source (electricity) to ensure that the cameras and lighting for camera imaging are efficient. The other limitation of the system is that it does not have a reservation where the user can book a slot for their vehicle. 
On the other hand, there is the possibility of utilizing typical outdoor cameras to upgrade the exactness of detection through the training of the algorithm with the images taken in various weather conditions, as expressed by Masmoudi et al. [50]. These researchers noted the solutions that incorporate the storing of an image of the area that has been captured from a camera in a database together with the coordinates of every space in the parking lot. The solutions also cover the cases when making comparisons among the sorted images that are sent in real time to determine whether a parking space is occupied. In such an instance, it is important to consider that when video cameras are used outside, efficacy can be lowered as a result of weather conditions, even when the training phase period with the images of various weather conditions has been completed. When it comes to the indoor parking lot, it can be a viable alternative considering that images are not impacted by weather conditions. Images are kept in a database for as long as it takes for the coordinates of every one of the themes to be compared to the images that are sent in real time in order to determine the availability of spaces [51].

A different approach based on visual information is proposed and developed in the already cited work of Alam et al. [32]. The authors introduce a new intelligent device equipped with an imaging sensor and a wireless communication module to create a smart camera with on-board computing capabilities. Smart cameras can be connected to each other in an Internet of Things (IoT) fashion, forming a so-called smart camera network (SCN). Since smart cameras can perform some tasks related to scene analysis and understanding using their local resources directly on board, cameras in an SCN can exchange their computation outputs instead of routinely transmitting raw images or video streams. Therefore, since the computation output is generally lower in size than raw data, bandwidth requirements are relieved, making SCN a scalable solution viable for video monitoring of large areas, such as parking lots. Specifically, in their paper, the authors address this application by presenting a smart camera hardware prototype on which an ad hoc computer vision algorithm is deployed. The proposed algorithm is space-based and attempts to infer each pre-codified parking space's status by analyzing its visual features using a combination of edge, color, and contrast clues. The evolution of the original algorithm and further experimentations were reported in Moroni et al. [33] and the already cited Amato et al. [52].

RFD tags, which have been utilized in the identification of cars, are dissipated to the user when they enter the parking spot. With the short-range wireless communication technologies as mentioned herein, the existing intelligent parking systems have the demerit of a short life, which is better for the sensor mode, as there is limited signal coverage and a high deployment cost. To cut down on the power consumption and investment, it is quite easy for drivers to pay for parking via the NB-IoT modules and the third-party payment platforms, which are discussed in the work [53]. Barone et al. [54] argue that intelligent parking systems are developed on the basis of RFID. Any time a car parks or leaves the parking space, the genetic lop locates the action, and the RFD reader obtains the card ID. The information layer is then sent to the unit controller to update the status of the parking space.

\section{Research Methods in Literature}

Parking is a service that is quite dated in the transport industry and it is thought to have evolved specifically for different generations. The initial parking system, in which there were not many vehicles, was articulated through the annual space renting model. However, with time, and as the number of vehicles in cities and towns continued to increase, just as the number of urban dwellers, the need for urban planning became apparent. With this, one area that required special attention, in order to ensure that vehicles entering urban areas do not cause traffic congestions as well as increase the harmful impacts they have on the environment, was urban parking. For this reason, the concept of controlled parking was borne. First, before the emergence of the smart parking system, cities relied on electronic parking services that involved the use of parking meters that were not wholly automated. 
This created loop-holes in the collection of fees and the auditing process. However, with technological advancement, there has been a notable terminal evolution, which has seen the emergence of smart parking systems. The present smart parking system provides the automation of different parking services, allowing consumers to navigate the entire parking experience independently_from the parking occupancy status to ticketing, parking, and fee settlement.

Various mechanisms have been suggested by different authors for different kinds of data collection in the research to solve the present urban parking problems. In recent works, the majority of the studies on smart parking are entirely focused on the technical positions, i.e., system architecture and design [15], operational algorithms and models [55], and prototype designs [56]. Many of them are focused on the solution rather than the algorithms, software, systems, and the brief situation of the technology of the sensors. Such studies have examined the merits and demerits, but they fall short in the bid to overcome the problems, as their inspiration lies in the selected technique of developing a new parking system based on the Internet of Things.

Pham et al. [27] enlisted a novel algorithm as a way to increase the efficacy of the existing cloud-based smart parking system and builds a network architecture based on the Internet of Things technology. Their proposal on the system assists users to automatically locate a free parking space at the least cost based on new performance metrics in the calculation of user parking costs by considering the distance and the holistic number of fee places in each car park. The costs are encapsulated by offering solutions to finding available parking spaces at the request of the user and the service of suggesting a new car park if the existing one is fully occupied. The simulation shows that the algorithm assists in upgrading the probability of successful parking and lowers time wastage. Mainetti et al. [35] presented a sophisticated IoT-aware smart parking system on the basis of the joint use of various technologies that include RFID, WSN NFC, and Mobile. It is capable of collecting the environmental parameters and the information on the occupancy state of parking spaces in real time. As a way of reducing the entire system costs, the possibility of using the solar RFID tag as a car detection system was analyzed. The system allowed the drivers to access the nearest empty spaces and to make payment for parking via the use of a customized mobile application. Additionally, the software app was developed on the basis of the RESTful Java and Google Cloud messaging technologies that were installed on the CS to manage alert events. A proof of concept was produced $t$ as a way of demonstrating the proposed solution's capability in satisfying the real requirements of an innovative smart parking system, while preliminary examination of the solar tag usage investigated the capability of the proposed detection solution.

Ji et al. [36] presented the generic concept of the use of cloud-based intelligent car parking services in smart cities as an instrumental application that deploys the Internet of Things (IoT) paradigm. The correspondence of an IoT subsystem included a sensor layer, communication layer, and application layer. A high-level loop in the system architecture was outlined as a way of demonstrating the provision of car parking services with the proposed functions. A cloud-based intelligent parking system that could be used within universities was articulated along with the principles of design and execution. Wang and He [14] designed and implemented a prototype of a reservation-based smart parking system that would allow drivers to efficiently locate and reserve empty parking spaces. They could learn the parking status from the sensor networks that were deployed in the parking spaces where the reservation process was impacted by the changes in the physical parking status, and the drivers were unable to access the cyber physical system with their personal communication devices. The researchers also studied and compared the performances of the smart parking policies of the smart parking system. The research results portrayed the proposed parking legislation as a potential tool in the simplification of the operations of parking systems as well as in the alleviation of traffic congestion made by parking searches. 
Chatzigiannakis et al. [42] adopted elliptic curve cryptography as an alternative to the convenient public key cryptography that can link RSA. ECC was a potential candidate in the execution of the constrained gadgets where the supreme computational resources such as speed and memory were limited to the low power wireless communication protocols that were incorporated. This was due to the attainment of similar security levels with traditional cryptosystems through the use of smaller parameter sizes. They would provide such with the generic implementation of the ECC that would run on various host operating systems that include Contiki, TinyOS, iSenseOS, ScatterWeb, and Aduino. Additionally, they would run on smartphone platforms such as Android and iOS and they would also use Linuxbased systems as noted by Schmidt et al. [57]. The implementation would not be contained in any unique platform. As such, it would allow the individual execution to run natively on diversified networks. Chatzigiannakis et al. [42] looked into the smart parking application domain and provided solutions that would safeguard the confidentiality of consumers by entirely evading the exchange of personal information. They also discussed the ways to safeguard users' confidentiality through the adoption of the test of zero-knowledge proofs with ECC execution. Their study also examined the performance of the system in a real-world outdoor IoT test bed and the analysis of the execution time and the network overhead for the available hardware stage. According to them, the codes are as transparent as open-source software, and they can be utilized by developers who aim to achieve high levels of security and privacy in the applications.

\section{Technical Analysis of the Literature}

Vehicle creation has developed impressively over the past 30 years, as discussed in [58]. More vehicles on the roads causes more fuel and time utilization and a developing interest in parking spots. These issues can be tended to by advanced stopping arrangements, which are perhaps the most well-known use cases in the concept of the smart city and are employed to improve the quality of the life pattern of a city [59].

The engineering of advanced stopping arrangements is chiefly addressed by three components: sensors, organizing conventions, and programming arrangements. Sensors are the main component as they gather data and feed the entire framework. Systems administration conventions are represented by an entryway that carries out remote IoT conventions and interfaces sensors to the product frameworks. Finally, programming arrangements guarantee that data are accessible to all users through some kind of administration. For example, individuals can utilize these data to observe heat guides of zones with the most elevated stopping space inhabitance [60].

To carry out an advanced stopping arrangement, a few innovative segments are included, such as sensors, an organizing framework, and programming arrangements. With respect to stopping models, there are a few works that have been introduced by industry and established researchers. Some of them are centered around arrangement, while others focus on the calculations, programming, or frameworks, and some works discuss the innovation of the sensors. For example, the creators of [61] propose a methodology dependent on computerized reasoning (specialists) to identify accessible spots. In [62], the authors examine various ways to carry out smart parking arrangements, and they consider the entire environment of such kinds of arrangements, which essentially includes sensors, door choice, edge preparing, and server farm examination. Furthermore, the authors of [63] portray a design that is completely dependent on ZigBee innovation. In addition, the authors of [64] recommend man-made consciousness for advancing park search; however, they do not determine the specialized subtleties of execution, for example, explicit conventions or sensor types. Moreover, the work proposed in [65] shows the utilization of Bluetooth low energy (BLE) as a convention for associating sensors and passages. Bluetooth is a remote convention that upholds the association between end-gadgets. The BLE rendition does not burn through much energy and is important for remote IoT stack convention. Different arrangements, similar to those in [66], propose the utilization of IR sensors for engineering. Cell phones are likewise thought to be in these arrangements, especially to 
discover accessible spaces. Considering the previously mentioned study, it can be seen that there are no global norms or base models characterized for the execution of smart stopping frameworks. Along these lines, it is just as important to examine how various parts are being utilized as it is to distinguish propensities concerning their utilization in order to carry out a smart stopping arrangement.

Advanced stopping arrangements were created with numerous innovations and approaches; subsequently, a grouping was performed on the basis of the setup focuses. For this situation, three alternate points of view were chosen: sensors, the network framework, and administration given to clients. The previously mentioned points of view were chosen depending on the significance given in [67].

\subsection{Types of Sensors}

With respect to arrangement and traffic executives, the most important subject is parking [68]. Recently, making decisions regarding the accessibility of stopping is difficult if there are no components that permit one to distinguish on the off chance whether a parking space is empty or not. The aim of the sensors is to tackle this issue by sensing accessibility and informing the stopping framework through an organization entryway. However, while the sensors take care of the location issue, an enormous number of these are needed to practice satisfactory monitoring of a given space. There are sensors that do not cover enormous spaces, and, as such, one for each spot is required. In this way, the bigger the space, the more noteworthy the amount and, thus, the greater the expense. Note that for the sensors that are utilized, they require a mechanical framework for the vehicle that the information regards. This suggests the establishment of both cell- and passage-based information networks [69].

Similarly, in situations where remote correspondence is not achievable, an organized link to deal with the gathered data should be set up. Climate conditions are a constraint that should be considered and upheld by sensors. Despite the fact that there are choices for utilizing different sensors, like cell phones or cameras in enormous spaces, there are security worries that should be tended to first [70].

Sensors are the main element of a smart stopping framework as they feed the framework with important information. In this way, they must provide unwavering quality and require next to no or nearly no support. Sensors characterize organization innovation and the instrument for sending information to the strike (savvy stopping framework). They ought not rely upon human association in the provision of climate data. Energy utilization should be insignificant and, if conceivable, have its own self-supporting resource of energy (i.e., solar energy). Surely, the incorporation of microelectromechanical system (MEMS) sensors will help diminish size, power utilization, and cost and expand execution and lifetime [71]. This advancement will prompt sensors with various capacities as opposed to specific types.

Data recovery is quite possibly the main aspect of savvy stopping arrangements, and for this interaction, an extraordinary assortment of sensors offered in the market can be utilized. Among the various kinds of sensors for savvy leaving, the most well-known ones are ultrasonic sensors, magnetometers, cameras (utilized for recognizing vehicles and free spaces), cell sensors, and radars [72]. A summary of the kinds of sensors utilized in the surveyed papers is shown in Table 1.

This table is organized as follows. In the first column, paper references are given. The following columns display a wide range of sensors recognized during the survey. Any paper that utilizes a kind of sensor is marked with a dark circle "*" under any segment that applies; "-" represents something else being used. The section "Other" regards sensors that are not plainly indicated in the examined paper. The accompanying table contains papers that detail the utilization of any sensor from the examination performed. Generally, it can be observed that among established researchers, the most utilized sensors are ultrasonic sensors, while cameras and cell phones (accelerometers, gyrators, and magnetometers) are in second and third place, respectively. This can be credited to the way that ultrasonic 
sensors can distinguish with more noteworthy exactness the profundity and thickness of surfaces, as well as working at high recurrence and having high affectability and high force.

Table 1. Sensor classification.

\begin{tabular}{|c|c|c|c|c|c|c|c|}
\hline Reference & Camera & Ultrasonic & $\begin{array}{l}\text { Cellular } \\
\text { Sensors }\end{array}$ & Infrared & Radar & Other & Magnotemeter \\
\hline 15 & - & * & - & * & - & - & - \\
\hline 18 & - & - & * & - & - & - & - \\
\hline 21 & - & * & - & - & - & - & - \\
\hline 24 & * & - & - & - & - & - & - \\
\hline 27 & - & * & - & - & * & - & - \\
\hline 30 & - & - & - & * & - & $*$ & - \\
\hline 33 & - & - & * & - & - & - & - \\
\hline 35 & - & * & - & - & - & - & - \\
\hline 36 & - & - & - & * & - & - & - \\
\hline 38 & - & - & - & - & - & * & - \\
\hline 41 & - & - & - & * & - & - & - \\
\hline 43 & - & * & - & - & - & - & - \\
\hline 45 & $*$ & - & - & * & - & - & - \\
\hline 46 & - & - & - & - & * & - & - \\
\hline 51 & - & - & * & - & - & - & - \\
\hline 56 & - & - & - & * & - & - & - \\
\hline 57 & - & * & - & - & - & $*$ & - \\
\hline 59 & * & - & - & - & - & - & - \\
\hline
\end{tabular}

The order recorded in the table above shows that sensors are generally not used in combination to deliver advanced stopping arrangements. A few recommendations, similar to those in [73], do not determine a particular type of sensor; however, the developers expect that a vehicle may contain cameras, ultrasonic sensors, and radar sensors. Different methodologies, such as that presented in [74], determine that the TSOP 1738 infrared sensor is accountable for identifying the presence or nonappearance of a vehicle. All things considered, the combination of sensors will surely deliver quality arrangement since the information will come from numerous sources that have various merits. The sensor in each audited work is utilized to cover the need to assemble data relying upon the attributes of the climate. A concise explanation of how sensors are utilized is portrayed below.

\subsection{Systems Administration}

Systems administration conventions are critical to pass data from sensors to advanced stopping framework; without them, it is difficult for each sensor setup to recover its information. In advanced stopping arrangements, there are two kinds of organization conventions (one for clients and one for sensors). At times, sensors and clients may utilize a similar convention. Nonetheless, client conventions consume more power and require an Internet network. However, most sensor conventions do not interface straightforwardly with the Internet; they need to go through an entryway at times (i.e., LoRa, ZigBee, and NB-IoT). This door is accountable for interpreting a wireless IoT convention to one that is TCP/IP based. The transmission of smart stopping arrangement requires an organization engineering framework to help in the communication associated with countless gadget at each specific time. Thus, considerations should be made regarding short-range and long-range correspondences for associating sensors with passages and afterward with programming arrangements. These organization executions need to focus on sending 
remote IoT conventions and cross-section organizations to cover more extensive spaces and permit sensors to communicate regardless of whether the door hub fizzles.

Systems administration in smart stopping must be adjusted to low energy utilization, minimal postponement, and dependable throughput. There are activities in certain areas to improve the organization of smart stopping, as portrayed in [75].

The utilization of a few correspondence arrangements has been proposed to be insufficient in some exploration papers. In this specific circumstance, the arrangements were ordered into two significant classes, as demonstrated in Table 2. The "sensor organization" class, which is deemed the matter of most importance, portrays network engineering and conventions applied to sensor correspondence. At this point, the "client network" classification depicts conventions used to send valuable data to the end client. This characterization and the observable pattern in the utilization of remote organization advancements for sensor correspondence are depicted in [76]. Finally, the "vehicle network" class was not considered due to the absence of exploration papers containing data on such organizations; thus, this classification was excluded.

Table 2. Sensor network and user network classification.

\begin{tabular}{|c|c|c|c|c|c|c|c|}
\hline Reference & Wireless IoT Protocol & Specific Protocol & WiFi & $3 \mathrm{G} / 4 \mathrm{G}$ & Bluetooth & Wired & Not Defined \\
\hline 15 & & & & * & & & \\
\hline 18 & & & * & & & & \\
\hline 21 & & ZigBee & & & & & \\
\hline 24 & * & & & & & & \\
\hline 27 & & & & & * & & \\
\hline 30 & & & & * & & * & \\
\hline 33 & & & * & & & & \\
\hline 35 & & ZigBee & & & & & * \\
\hline 36 & & & & * & & & \\
\hline 38 & & IEEE 802.15.4 & & & & * & \\
\hline 41 & & & & * & & & \\
\hline 43 & & & & & & & * \\
\hline 45 & $*$ & & & * & & & \\
\hline 46 & & ZigBee (XBee) & & & * & & \\
\hline 51 & & & $*$ & & & & \\
\hline 56 & & & & * & & & * \\
\hline 57 & & & & & & * & \\
\hline 59 & $*$ & & & & & & \\
\hline
\end{tabular}

\subsubsection{Sensor Network}

This classification focuses on the most utilized advances for the sending of uses. The most significant subcategories are wireless conventions (LPWAN and LR-WPAN conventions, such as LoRa, NB-IoT, and ZigBee), Wi-Fi, and cellular innovations (3G/4G). Other subcategories incorporate Bluetooth and other wired advancements (i.e., Ethernet, USB, and sequential interchanges).

Table 2 contains two significant sections-sensor organization and client organizationwhich address the type of systems administration innovation utilized by a sensor or client to interact with the foundation of a smart parking solution. These sections were classified into different levels of systems administration availability. Remote convention suggests 
that a remote IoT convention is portrayed in the examined paper; assuming that this is the case, the "particular protocol" section includes the name of the pre-owned convention.

If a paper coordinates with a particular section, "**" appears, while in all other cases, there is a clear space. Only the papers that predetermined a kind of systems administration innovation are considered in this table.

\subsubsection{Client Network}

Client network arrangement is divided into four subcategories. The principal classification is called "any innovation that empowers Web access", but research papers did not discuss the conventions utilized but suggested that any gadget with Web access could obtain information from a framework. The second and third classes involve "just Wi-Fi" and "just cellular advances $(3 \mathrm{G} / 4 \mathrm{G})$ ", respectively. The last class covers wired innovations that can be Ethernet, USB, and sequential correspondences, among others. Every one of the papers evaluated in this classification is ordered by the previously discussed subcategories. Such characterization is shown in Table 2. From Table 2, it can be clearly seen that wireless IoT protocols are for the most part utilized in local research areas for the association of sensors with doors together the mix of $\mathrm{Wi}-\mathrm{Fi}$ and $3 \mathrm{G} / 4 \mathrm{G}$ to send data over to a TCP/IP organization. However, for the user network, the realized advancements are utilized to associate, oversee, or consume data from smart stopping arrangements.

\section{Discussion}

Traditional systems use loop detectors in the entry and exit points in the tracking process of parking availability. However, the new smart parking system needs the installation of wireless sensors in individual, single parking on streets [77]. As IoT technology evolves, there are no established coordinators that monitor the creation and proposition of the various solutions and standards. It has been observed that in such instances, there will be some loop-holes in the forthcoming years and even in the later stages of heterogeneity [22]. There is a wide range of seminorms involved in the subject of IoT, which cover various views and, therefore, address the sectorial ideologies that suggest that universes do not argue with themselves. Various communication solutions are utilized in the device model [78] to overcome issues that are normally inherent to a single point of failure. Issues such as susceptibility to the distributed lack of service attack and the cases of distant hijacking attacks are capable of making parking unavailable. Furthermore, there are issues that expose the sensitive information belonging to drivers and their parking information, which is stored in the database due to the risk of privacy breach and loss. The large number of interconnected devices gives rise to the scaling issues and the flexible infrastructure that is needed to deal with the security threat in an effectual surrounding [79].

When there are no application layers, the smart parking system is limited in the performance of primitive tasks. The application layer has to make provision for the real-time information, which assists motorists in making appropriate decisions. The architecture has to be somewhat compact such that it can deal with the massive amount of information and the provision of services to a large scale of users. As a way of achieving this, there is a need to deploy cloud infrastructures in the public or in the private sphere. The data could show the sections that need a high concentration and suggest an alternative for consumers who are nearby. Additionally, the data could be used to make parking predictions and provide information regarding the availability of spaces in areas where there are no sensors and there is poor communication coverage. Commercially, the perspective of this information could be of significance as a service point that establishes the nearby sites where there is high vehicular congestion. Furthermore, construction companies can benefit from the information derived from the analysis of data on different parking aspects, especially when determining the places where they can construct more parking lots and increase the number of parking spaces. 


\section{Future Directions}

The general plans of action for smart parking fall into two general classes: horizontal plans, concerning the empowering segments and innovation, and vertical plans, which coordinate these advancements to supply an end client with an offer. The former plans of action are superior and less expensive detection innovations than the latter. The second plans of action place importance on stopping frameworks regarding information, social occasions, stockpiling, and curation. The third-level plan of action regards scientific strategies to transform the information assembled into noteworthy data. While the initial two strategies have been the focus of previous research, Parking 4.0 will open doors in the third section. Stopping is required to develop a smart and green industry with commoditization of new advances (particularly in IoT/M2M/V2X1 space) and with the appearance of new eco-framework players and members. It is expected that early certain progressions will (and are starting to) occur with the following:

- Electric vehicles, which add another measurement to in determining how quickly cars leave their parking spaces, with charging credits (such as accessibility of charging stations, time and charging term, and evaluation and energy markets) becoming a key factor.

- Autonomous vehicles, which will generally change the use of vehicles and how they leave parking spaces through self-leaving abilities and mechanical valets.

- Uberization 2.0 of stopping, which will make an exceptionally receptive and ongoing eco-framework, associating parties interested in loaning parking spots with those looking for one. As the bigger leaving eco-framework develops into incorporated software, we anticipate a significant number of standard tasks (as those illustrated above) to relocate into in-vehicle frameworks with leaving application stores, there by easing the arrangement weight of leaving frameworks (both on and off road).

This could be increased with omnipresent or quickly developing versatility foundations (such as the electronic cost assortment/ETC 3 transponders) to accomplish the framework scale and densities at moderate expenses as opposed to depending on a huge transmission of custom sensor networks [80]. Moreover, adaptation motors and expediting administrations that help interface information buyers with information makers could be empowered, utilizing the structure squares of information proprietorship or motivation instruments through acknowledgment and attribution, bargains, or financial offers. Similarly, as with all new advancement ideal models, such prospects accompany their own exploration challenges.

\section{Conclusions}

The smart parking system can be realized as a means to solve parking issues both in the current era and in the future. Furthermore, IoT-enabling techniques need to be given maximum attention, ensuring that they are at the center of planning smart parking systems. In line with this, there exist several alternatives to the mechanism of modernizing the setup of parking lots and to the implementation of smart functionality. There are those that can be efficiently installed and there are those that are quite challenging. Regardless, the implementation has to allow drivers to acquire actual information on parking online and on the remaining parking spaces. The parking process in the city has to be addressed fully-in an efficient, real-time, and cost-effective manner.

Technology and parking center companies need to team up to provide the muchrequired parking solution and to assist drivers in saving time and energy. The combined effort can also provide priceless and valuable analytics to malls, shop owners, and federal institutions in regard to planning of affairs, especially in relation to parking spaces. For federal institutions, the adoption of a smart parking system could give them a head start as the network can detect parking rules violation, register them, and then collect and store the required evidence. Similarly, due to automation, the network can issue a ticket and then make a notification to the violating party in seconds. 
The existing parking services are unique to a given locality. Parking is marked by the perimeters of the confined facilities, and there are no possibilities or mechanisms of allowing clients to park in any other collocated garages or via any consolidated mechanism. Whereas the model provides simplicity in the operations, there is a missed opportunity for individuals to have good parking experiences. Parking operators need to expand their revenue outlets by capitalizing on the possibilities of adopting the consolidated services that smart parking systems are making possible.

The future of the smart parking system is projected to be promising, as these are tied to technologies such as the IoT, artificial intelligence, machine learning, augmented reality, and other advanced modern technologies. These are the same technologies that are driving digital transformation for businesses in this era of the fourth industrial revolution. The leverages of innovation and future smart parking systems will improve parking system efficiencies by solving the issues that occur as a result of urbanization. For instance, there is some interest in the ideology of using parking lifts in the management of parking. This is a mechanical process, where a car is stacked on the available overhead space, thereby ensuring that more than one car can occupy the same parking space by being stacked above or below each other in two, three, or more layers. As such, the future parking system may be a smart parking system that integrates different players in different industries as well as the provision of diverse services. This way, existing parking practices can be accommodated for, and the transition to modern, smart parking systems can be realized.

Author Contributions: Methodology, Z.A.; investigation, G.P.; resources, D.M.; writing, C.B.; supervision, S.O.; project administration, E.O.; writing-review and editing, M.O.; formal analysis, M.K. All authors have read and agreed to the published version of the manuscript.

Funding: This research received no external funding.

Institutional Review Board Statement: Not applicable.

Informed Consent Statement: Not applicable.

Data Availability Statement: Not applicable.

Conflicts of Interest: The authors declare no conflict of interest.

\section{References}

1. IIHS-IHLD (Insurance Institute for Highway Safety: Highway Loss Data), 2019, Fatality Facts 2018 Urban/rural Comparision (WWW Document). Available online: https:/ /www.iihs.org/topics/fatality-statistics/detail/urban-rural-comparision (accessed on 27 April 2021).

2. Tidey, A. Road fatalities: Which EU Countries Are the Most Dangerous? Available online: https://www.euronews.com/2019/0 8/20/road-fatalities-which-eu-countries-are-the-most-dangerous (accessed on 9 January 2021).

3. Gössling, S. Why cities need to take road space from cars-and how this could be done. J. Urban Des. 2020, 25, 443-448. [CrossRef]

4. Parkopedia. Global Parking Index. 2019. Available online: https://cdn2.hubspot.net/hubfs/5540406/Parkopedia-GlobalParking-Report-2019_FINAL.pdf?_hstc=91933061.ccb59d1927f24372f8e1e69fd80e8c78.1569194984492.1569194984492.156919 4984492.1\&_hssc=91933061.1.1569194984493\#: \{\}:text=In\%202019\%2C\%20New\%20York\%20maintains, prices\%2C\%20from\% 20\%2432.97\%20to\%20\%2434.94 (accessed on 9 January 2021).

5. Fraifer, M. A Design Exploration of An IoT Based Smart Parking System with Stakeholders Using User-Centred Design. Ph.D. Thesis, University of Limerick, Limerick, Ireland, 2018.

6. Walsh, D. Reducing Our Reliance on Cars: The Shifting Future of Urban Transportation. Available online: https:/ /mitsloan.mit. edu/ideas-made-to-matter/reducing-our-reliance-cars-shifting-future-urban-transportation (accessed on 11 January 2021).

7. Jaller, M.; Holguín-Veras, J.; Hodge, S.D. Parking in the City: Challenges for Freight Traffic. Transp. Res. Rec. 2013, 2379, 46-56. [CrossRef]

8. Hasenfuss, H.; Fraifer, M.; Kharel, S.; Elmangoush, A.; Ryan, A.; Elgenaidi, W. "It takes two to tango: Merging science and creativity to support continued innovation in the IoT domain". Special Issue/Section: Special Issue on Multidisciplinary Sciences and Engineering. Adv. Sci. Technol. Eng. Syst. J. 2018, 2, 82-91.

9. Brown, L.R. Redesigning Cities for People: Car-Centered Urban Sprawl. In Eco-Economy: Building an Economy for the Earth; Earthscan: Washington, DC, USA, 2003.

10. Adler, J.L.; Satapathy, G.; Manikonda, V.; Bowles, B.; Blue, V.J. A multi-agent approach to cooperative traffic management and route guidance. Transp. Res. Part B Methodol. 2005, 39, 297-318. [CrossRef] 
11. Naphade, M.; Banavar, G.; Harrison, C.; Paraszczak, J.; Morris, R. Smarter Cities and Their Innovation Challenges. Computer 2011, 44, 32-39. [CrossRef]

12. Babic, M.; Vekj, A.; Stanojevic, M.; Ostojic, G.; Borocki, J.; Stankovski, S. Modern Parking Solutions for Smart Cities. In Proceedings of the 30th DAAAM International Symposiu on Intelligent Manufacturing and Automation, Zadar, Croatia, 23-26 October 2019.

13. Borgonovo, E.; Gatti, S.; Peccati, L. What drives value creation in investment projects? An application of sensitivity analysis to project finance transactions. Eur. J. Oper. Res. 2010, 205, 227-236. [CrossRef]

14. Wang, H.; He, W. A Reservation-based Smart Parking System. In Proceedings of the Conference on Computer Communications Workshops, Shanghai, China, 10-15 April 2011.

15. Kuran, M.Ş.; Viana, A.C.; Iannone, L.; Kofman, D.; Mermoud, G.; Vasseur, J.P. A Smart Parking Lot Management System for Scheduling the Recharging of Electric Vehicles. IEEE Trans. Smart Grid 2015, 6, 2942-2953. [CrossRef]

16. Sajeev, A.; Vidwans, S.; Mallick, C.; Jog, Y. Understanding Smart and Automated Parking Technology. Int. J. Serv. Sci. Technol. 2015, 8, 251-262.

17. Parmar, J.; Das, P.; Dave, S.M. Study on demand and characteristics of parking system in urban areas: A review. J. Traffic Transp. Eng. 2020, 7, 111-124. [CrossRef]

18. Nižetić, S.; Šolić, P.; López-de-Ipiña González-de-Artaza, D.; Patrono, L. Internet of Things (IoT): Opportunities, issues and challenges towards a smart and sustainable future. J. Clean. Prod. 2020, 274, 122877. [CrossRef]

19. Khanna, A.; Anand, R. IoT based smart parking system. In Proceedings of the International Conference on Internet of Things and Applications (IOTA), Pune, India, 25-30 November 2016.

20. Patil, M.; Bhonge, V.N. Wireless Sensor Network and RFID for Smart Parking System. Int. J. Emerg. Technol. Adv. Eng. 2013, 3, 188-192.

21. Kayal, P.; Perros, H. A comparison of IoT application layer protocols through a smart parking implementation. In Proceedings of the 20th Conference on Innovations in Clouds, Internet and Networks (ICIN), Paris, France, 7-9 March 2017.

22. Soaibuzzaman, A.S.; Rahman, M.S.; Rahaman, M. A Blockchain-Based Architecture for Integrated Smart Parking Systems. In Proceedings of the International Conference on Pervasive Computing and Communications Workshops, Kyoto, Japan, 11-15 March 2019.

23. Yang, J.; Portilla, J.; Riesgo, T. Smart parking service based on Wireless Sensor Networks. In Proceedings of the 38th Annual Conference on IEEE Industrial Electronics Society, Montreal, QC, Canada, 25-28 October 2012.

24. Allam, Z. On Smart Contracts and Organisational Performance: A Review of Smart Contracts through the Blockchain Technology. Rev. Econ. Bus. Stud. 2018, 11, 137-156. [CrossRef]

25. Bakici, T.; Almirall, E.; Wareham, J. A Smart City Initiative: The Case of Barcelona. J. Knowl. Econ. 2012, 4, 135-148. [CrossRef]

26. Sotres, P.; Lanza, J.; Sánchez, L.; Santana, J.R.; López, C.; Muñoz, L. Breaking Vendors and City Locks through a Semantic-enabled Global Interoperable Internet-of-Things System: A Smart Parking Case. Sensors 2019, 19, 229. [CrossRef]

27. Pham, N.; Hassan, M.; Nguyen, H.M.; Kim, D. GS1 Global Smart Parking System: One Architecture to Unify Them All. In Proceedings of the International Conference on Services Computing (SCC), Honolulu, HI, USA, 25-30 June 2017.

28. Zacepins, A.; Komasilovs, V.; Kviesis, A.; Gatins, A.; Skudra, M.; Pierhurovics, A. Implementation of Smart Parking System in Jelgava City in Latvia. In Proceedings of the 11th International Conference on Application of Information and Communication Technologies (AICT), Moscow, Russia, 20-22 September 2017.

29. de la Torre, C.L.; Sotres, P.; Sánchez, L.; Jeong, S.M.; Kim, J. Smart City Services Over a Global Interoperable Internet-ofThings System:The Smart Parking Case. In Proceedings of the 2018 Global Internet of Things Summit (GIoTS), Bilbao, Spain, 4-7 June 2018.

30. Lanza, J.; Sánchez, L.; Gutierrez, V.; Galache, J.; Santana, J.; Sotres, P.; Muñoz, L. Smart City Services over a Future Internet Platform Based on Internet of Things and Cloud: The Smart Parking Case. Energies 2016, 9, 719. [CrossRef]

31. Leone, G.R.; Moroni, D.; Pieri, G.; Petracca, M.; Salvetti, O.; Azzarà, A.; Marino, F. An intelligent cooperative visual sensor network for urban mobility. Sensors 2017, 17, 2588. [CrossRef]

32. Alam, M.; Moroni, D.; Pieri, G.; Tampucci, M.; Gomes, M.; Fonseca, J.; Leone, G.R. Real-time smart parking systems integration in distributed ITS for smart cities. J. Adv. Transp. 2018, 2018. [CrossRef]

33. Moroni, D.; Pieri, G.; Leone, G.R.; Tampucci, M. Smart cities monitoring through wireless smart cameras. In Proceedings of the 2nd International Conference on Applications of Intelligent Systems, Kuala Lumpur, Malaysia, 20-21 March 2019; pp. 1-6.

34. Tsai, M.; Pham, T.N.; Nguyen, D.B.; Dow, C.; Deng, D. A Cloud-Based Smart-Parking System Based on Internet-of-Things Technologies. IEEE Access 2015, 3, 1581-1591.

35. Mainetti, L.; Marasovic, I.; Patrono, L.; Solic, P.; Stefanizzi, M.L.; Vergallo, R. A Novel IoT-aware Smart Parking System based on the integration of RFID and WSN technologies. Int. J. RF Technol. 2016, 7, 175-199. [CrossRef]

36. Ji, Z.; Ganchev, I.; O’Droma, M.; Zhao, L.; Zhang, X. A cloud-based car parking middleware for IoT-based smart cities: Design and implementation. Sensors 2014, 14, 22372-22393.

37. Kotb, A.O.; Shen, Y.; Huang, Y. Smart Parking Guidance, Monitoring and Reservations: A Review. IEEE Intell. Transp. Syst. Mag. 2017, 9, 6-16. [CrossRef]

38. Tomar, P.; Kaur, G.; Singh, P. A Prototype of IoT-Based Real Time Smart Street Parking System for Smart Cities. In Internet of Things and Big Data Analytics Toward Next-Generation Intelligence. Studies in Big Data; Springer: Cham, Switzerland, 2018; Volume 30, pp. 243-263. 
39. Shi, J.; Jin, L.; Li, J.; Fang, Z. A smart parking system based on NB-IoT and third-party payment platform. In Proceedings of the 17th International Symposium on Communications and Information Technologies (ISCIT), Cairns, QLD, Australia, 25-27 September 2017.

40. Shelby, Z.; Bormann, C. 6LoWPAN: The Wireless Embedded Internet; John Wiley \& Sons: Hoboken, NJ, USA, 2011 ; Volume 43.

41. Alessandrelli, D.; Petracca, M.; Pagano, P. T-res: Enabling reconfigurable in-network processing in IoT-based wsns. In Proceedings of the International Conference on Distributed Computing in Sensor Systems, Cambridge, MA, USA, 20-23 May 2013; pp. 337-344.

42. Chatzigiannakis, I.; Vitaletti, A.; Pyrgelis, A. A privacy-preserving smart parking system using an IoT elliptic curve based security platform. Comput. Commun. 2016, 89, 165-177. [CrossRef]

43. Yaqoob, I.; Ahmed, E.; Rehman, M.H.U.; Ahmed, A.I.A.; Al-garadi, M.A.; Imran, M.; Guizani, M. The rise of ransomware and emerging security challenges in the Internet of Things. Comput. Netw. 2017, 129, 444-458. [CrossRef]

44. Chauhan, V.; Patel, M.; Tanwar, S.; Tyagi, S.; Kumar, N. IoT Enabled real-Time urban transport management system. Comput. Electr. Eng. 2020, 86, 106746. [CrossRef]

45. Martinez-Balleste, A.; Perez-Martinez, P.; Solanas, A. The Pursuit of Citizens' Privacy: A privacy-Aware Smart City is Possible. IEEE Commun. Manag. 2013, 51, 136-141. [CrossRef]

46. Zoonen, L.V. Privacy concerns in smart cities. Gov. Inf. Q. 2016, 33, 472-480. [CrossRef]

47. Pramanik, P.K.D.; Upadhyaya, B.K.; Pal, S.; Pal, T. Chapter 1-Internet of things, smart sensors, and pervasive systems: Enabling connected and pervasive healthcare. In Healthcare Data Analytics and Management; Academic Press: Cambridge, MA, USA, 2019; pp. $1-58$.

48. Postigo, C.G.D.; Torres, J.; Menéndez, J.M. Vacant parking area estimation through background subtraction and transience map analysis. IET Intell. Transp. Syst. 2015, 9, 835-841. Available online: https://digital-library.theiet.org/content/journals/10.1049/ iet-its.2014.0090 (accessed on 6 February 2021). [CrossRef]

49. Fraifer, M.; Fernström, M. Investigation of Smart Parking Systems and their technologies. In Proceedings of the Thirty Seventh International Conference on Information Systems, IoT Smart City Challenges Applications, Dublin, Ireland, 9 December 2016.

50. Masmoudi, I.; Wali, A.; Jamoussi, A.; Alimi, A.M. Vision based system for Vacant Parking Lot Detection: VPLD. In Proceedings of the International Conference on Computer Vision Theory and Applications (VISAPP), Lisbon, Portugal, 5-8 January 2014.

51. Shih, S.; Tsai, W. A Convenient Vision-Based System for Automatic Detection of Parking Spaces in Indoor Parking Lots Using Wide-Angle Cameras. IEEE Trans. Veh. Technol. 2014, 63, 2521-2532. [CrossRef]

52. Amato, G.; Bolettieri, P.; Moroni, D.; Carrara, F.; Ciampi, L.; Pieri, G.; Vairo, C. A wireless smart camera network for parking monitoring. In Proceedings of the Globecom Workshops, Abu Dhabi, United Arab Emirates, 9-13 December 2018; pp. 1-6.

53. Rashid, B.; Rehmani, M.H. Applications of wireless sensor networks for urban areas: A survey. J. Netw. Comput. Appl. 2016, 60, 192-219. [CrossRef]

54. Barone, R.; Giuffrè, T.; Siniscalchi, M.; Morgano, M.; Tesoriere, G. Architecture for parking management in smart cities. Inst. Eng. Technol. 2013, 8, 1-8. [CrossRef]

55. Caicedo, F.; Blazquez, C.; Miranda, P. Prediction of parking space availability in real time. Expert Syst. Appl. 2012, 39, 7281-7290. [CrossRef]

56. Márquez, M.D.; Lara, R.A.; Gordillo, R.X. A new prototype of smart parking using wireless sensor networks. In Proceedings of the Colombian Conference on Communications and Computing (COLCOM), Bogota, Colombia, 4-6 June 2014.

57. Schmidt, A.; Schmidt, H.; Batyuk, L.; Clausen, J.H.; Camtepe, S.A.; Albayrak, S.; Yildizli, C. Smartphone malware evolution revisited: Android next target? In Proceedings of the 4th International Conference on Malicious and Unwanted Software (MALWARE), Montreal, QC, Canada, 13-14 October 2009.

58. Li, M.; Lu, J.; Chen, Z.; Amine, K. 30 years of lithium-ion batteries. Adv. Mater. 2008, 30, 1800561.

59. Pearre, N.S.; Kempton, W.; Guensler, R.L.; Elango, V.V. Electric vehicles: How much range is required for a day's driving? Transp. Res. Part C Emerg. Technol. 2011, 19, 1171-1184. [CrossRef]

60. Tayyaba, S.; Khan, S.A.; Ashraf, M.W.; Balas, V.E. Home Automation Using IoT. In Recent Trends and Advances in Artificial Intelligence and Internet of Things; Springer: Cham, Switzerland, 2020; pp. 343-388.

61. Seaver, N. Captivating algorithms: Recommender systems as traps. J. Mater. Cult. 2019, 24, 421-436. [CrossRef]

62. Ge, Y.; Zhang, X.; Han, B. Complex IoT control system modeling from perspectives of environment perception and information security. Mob. Netw. Appl. 2017, 22, 683-691.

63. Ravitej, K.S.; Sukumar, M.M.; Felix, A.Y. Multimode children tracking-Using ZigBee device. In Proceedings of the International Conference on Computation of Power, Energy Information and Commuincation, Melmaruvathur, India, 22-23 March 2017; pp. 691-694.

64. Johnson, S. Emergence: The Connected Lives of Ants, Brains, Cities, and Software; Simon and Schuster: New York, NY, USA, 2002.

65. Nagaraj, A. Introduction to Sensors in IoT and Cloud Computing Applications; Bentham Science Publishers: Sharjah, United Arab Emirates, 2021.

66. Atiqur, R. Smart car parking system model for urban areas. Comput. Sci. Inf. Technol. 2021, 2, 95-102. [CrossRef]

67. Komninos, N. The Age of Intelligent Cities: Smart Environments and Innovation-For-All Strategies; Routledge: Oxfordshire, UK, 2014.

68. Giuffrè, T.; Siniscalchi, S.M.; Tesoriere, G. A novel architecture of parking management for smart cities. Procedia Soc. Behav. Sci. 2012, 53, 16-28. [CrossRef] 
69. Grocholsky, B.; Keller, J.; Kumar, V.; Pappas, G. Cooperative air and ground surveillance. IEEE Robot. Autom. Mag. 2006, 13, 16-25. [CrossRef]

70. Hossain, M.A. Framework for a cloud-based multimedia surveillance system. Int. J. Distrib. Sens. Netw. 2014, 10, 135257. [CrossRef]

71. Wang, B. Coverage Control in Sensor Networks; Springer Science \& Business Media: Berlin, Germany, 2010.

72. Yuen, S.C.Y.; Yaoyuneyong, G.; Johnson, E. Augmented reality: An overview and five directions for AR in education. J. Educ. Technol. Dev. Exch. 2011, 4, 11.

73. Knight, W. Driverless cars are further away than you think. Technol. Rev. 2013, 116, 44.

74. Barriga, J.J.; Sulca, J.; León, J.L.; Ulloa, A.; Portero, D.; Andrade, R.; Yoo, S.G. Smart parking: A literature review from the technological perspective. Appl. Sci. 2019, 9, 4569. [CrossRef]

75. Ali, M.T.; Rahim, S.S.; Jan, M.A.; Ishtiaq, A.; Ahmed, S.; Ahmad, M.; Khan, M.A. Dist-Coop: Distributed cooperative transmission in UWSNs using optimization congestion control and opportunistic routing. Int. J. Adv. Comput. Sci. Appl. $2018,9,356-368$.

76. Heumann, B.W. Satellite remote sensing of mangrove forests: Recent advances and future opportunities. Prog. Phys. Geogr. 2011, 35, 87-108. [CrossRef]

77. Geng, Y.; Cassandras, C.G. New "Smart Parking” System Based on Resource Allocation and Reservations. IEEE Trans. Intell. Transp. Syst. 2013, 14, 1129-1139. [CrossRef]

78. Bagula, A.; Castelli, L.; Zennaro, M. On the Design of Smart Parking Networks in the Smart Cities: An Optimal Sensor Placement Model. Sensors 2015, 15, 15443-15467. [CrossRef] [PubMed]

79. Abdulkader, O.; Bamhdi, A.M.; Thayananthan, V.; Jambi, K.; Alrasheedi, M. A novel and secure smart parking management system (SPMS) based on integration of WSN, RFID, and IoT. In Proceedings of the 15th Learning and Technology Conference (L\&T), Jeddah, Saudi Arabia, 25-26 February 2018.

80. Kuorilehto, M.; Hännikäinen, M.; Hämäläinen, T.D. A survey of application distribution in wireless sensor networks. EURASIP J. Wirel. Commun. Netw. 2005, 5, 1-15. [CrossRef] 